\title{
Raumplanerische Behandlung von Brachland
}

\section{Einleitung}

Das Phänomen «Brachland» bildet in ausgedehnten Gebieten, vorab des schweizerischen Berggebietes und der Voralpen ein bedeutendes Landschaftselement (SURBER 1979). Diese Feststellung allein vermag nicht zu genügen. Unweigerlich stellt man sich die Frage, was wohl die Zukunft dieser Gebiete sein wird. Von der Beschreibung dieses Phänomens Brachland führt diese Frage hinüber in die raumplanerische Behandlung von Brachland.

\section{Die Brachlandfrage als Bestandteil der Richtplanung}

Seit dem 1.1.1980 verfügen wir über das Bundesgesetz über die Raumplanung (RPG). Da es in diesem Artikel darum geht, die Brachlandfrage auf dem Hintergrund der Raumplanung zu beleuchten, sollen vorerst einige Erläuterungen zu diesem Gesetz gemacht werden:

Im RPG wird differenziert zwischen Richtplanung und Nutzungsplanung. Eine scharfe Abgrenzung dürfte schwierig sein, doch läßt sich als Annäherung formulieren, da $\beta$ sich Richtplanung mit der Vorbereitung von raumwirksamen Handlungen befaßt. Die Vorbereitung von Handlungen kennzeichnet sich durch verschiedene Stadien. Die Verordnung über die Raumplanung (VO RPG) vom 26. August 1981 unterscheidet folgerichtig zwischen dem Aufnehmen von Absichten über die künftige Nutzung des Bodens (Vororientierungen), dem Rapportieren über den Stand der Bearbeitung ungelöster Probleme (Zwischenergebnisse) und dem resultierenden Beschluß (Festsetzung) bezüglich der zu treffenden Handlungen. Die grundsätzliche Koordination und Disposition bezüglich der künftigen Nutzung des Bodens geschieht also auf der Ebene der Richtplanung. Die Nutzungsplanung auf der andern Seite umfaßt dann die Absprachen über die konkrete Realisierung eines Vorhabens.

In der Folge befassen wir uns ausschließlich mit der Ebene der Richtplanung, denn hier liegen die bedeutendsten Neuerungen des Raumplanungsgesetzes. Thematisch steht bei der Richtplanung die Behandlung ungelöster Probleme im Vordergrund. Aus den bisherigen Erfahrungen zeichnet sich ab, daß sinnvol- lerweise zwischen zwei Gruppen ungelöster Probleme unterschieden wird:

a) Probleme, die entstehen, weil für die Nutzung einer bestimmten Fläche mehrere sich ausschlieBende Absichten geäußert werden (räumliche Konflikte) oder weil

b) bisherige Nutzungen aufgegeben und nicht durch neue ersetzt werden. In diese zweite Gruppe fallen etwa Entwicklungen wie der Zerfall der Zentren großer Städte und fällt auch das hier zur Diskussion stehende Phänomen Brachland.

Diese Ausführungen erlauben eine erste Feststellung: Eine kantonale Richtplanung (in Gebieten, wo Brachland vorkommt), die keine Aussagen über Brachland macht, erfüllt den Auftrag zur Durchführung der Raumplanung gemäß dem geltenden Bundesgesetz nicht.

\section{Die Integration des Brachlandes in die Richtplanung}

Was heißt nun «Integration» des Phänomens Brachland in die Richtplanung?

Zur Klärung dieser Frage sind einige Präzisierungen zu den im Gesetz verwendeten Begriffen «Richtpläne» und «Richtplanung» nötig. Im erwähnten Gesetz wird eine klare Unterscheidung dieser beiden Begriffe vorgenommen:

Die Bearbeitung ungelöster Probleme geschieht im Rahmen der Richtplanung als Prozeß, während die Richtpläne die Beschlüsse, die aus der Bearbeitung resultieren, enthalten. Diese Beschlüsse sind als behördenverbindlich erklärt, um ihnen das nötige politische Gewicht zu geben. Beschlüsse in der Phase der Zwischenergebnisse beinhalten Aussagen darüber, wer mit wem bis wann über was zu verhandeln hat. Somit enthalten die Richtpläne nicht nur Lösungen von Problemen (Festsetzungen), sondern enthalten auch noch ungelöste Probleme, die aber in Bearbeitung sind (Zwischenergebnisse) und solche, die noch nicht bearbeitet werden (Vororientierungen).

Dr. Peter Gresch, ORL-Institut ETHZ, 8093 Zürich 
Die Beschlüsse, unabhängig auf welche Phase sie sich beziehen, müssen begründet sein. Die Begründungen ihrerseits müssen aus den Grundlagen ableitbar sein. Grundlagen spielen demzufolge auch in der modernen Richtplanung eine bedeutende Rolle.

Kehren wir nach diesem Exkurs wieder zum Problem Brachland zurück.

Nicht alles Brachland verlangt die gleiche Aufmerksamkeit. Aus einer raumplanerischen Beurteilung sind Anhaltspunkte für eine differenzierte Behandlung herauszuschälen.

$\mathrm{Zu}$ den Grundlagen einer derartigen Beurteilung gehören sicher folgende Übersichten:

- gegenwärtige Situation und

- mögliche künftige Entwicklung bezüglich Brachlegung.

Im Rahmen der Richtplanung ist dann zu prüfen, mit welchen Wirkungen bei der Wahl bestimmter Handlungen zu rechnen wäre (Szenarien), denn der Entscheid für bestimmte Handlungen muß im Wissen um die zu erwartenden Wirkungen geschehen. Die eruierten Wirkungen sind einer sorgfältigen Beurteilung zu unterziehen. Es geht dabei darum herauszufinden, in welchen Gebieten Maßnahmen nötig sind, um bestehende oder zu erwartende Gefahren zu beseitigen bzw. abzuwenden. In zweiter Priorität ist festzustellen, in welchen Gebieten Brachlegung zu verhindern ist, um andere Nutzungen zu ermöglichen (z. B. Skiabfahrten) oder andern Interessen Rechnung zu tragen (z. B. Erhaltung von Kulturlandschaften).

Eine eingehende Behandlung der einzelnen Schritte dieses Planungsprozesses ist zu finden in SCHERRER 1980.

Hier seien lediglich einige, im Zusammenhang mit der Richtplanung zentral erscheinende Gesichtspunkte hervorgehoben. Zeigt sich aufgrund entsprechender Grundlagen, da $\beta$ in einem Gebiet Brachland vorkommt, ist (auf kantonaler Ebene) ein Beschluß zu fassen, der sicherstellt, daß ein Verfahren zur Bearbeitung der anstehenden Probleme eingeleitet wird. Da es sich beim Brachland um ein verschiedene Sachbereiche übergreifendes Problem handelt, ist es denkbar, $\mathrm{da} ß$ die kantonale Planungsstelle, zusammen mit andern beteiligten Stellen, die Vorbereitung entsprechender Anträge zu Beschlüssen der kantonalen Exekutive oder Legislative trifft. Die raumplanerische Behandlung von Brachland ist demnach nicht ausschließliche Domäne der Raumplaner. Es ist eine Aufgabe, die gemeinsam durch alle Träger raumwirksamer Handlungen wahrzunehmen ist. Durch die Abstimmung ihrer Handlungen werden die stets begrenzten Mittel im Einsatz um die Lösung der anstehenden Brachlandprobleme in ihrer Wirkung gesteigert.

Primär wird es beim Brachland bei diesen Beschlüssen über künftige Nutzungen der betreffenden Flächen gehen. Diese Entscheide sind zu ergänzen durch Beschlüsse über flankierende Maßnahmen, wie Wegebau, Entwässerungen, Verbauungen, Güterzusam- menlegungen usw. Diese Maßnahmen sind umso nötiger, als eine Entscheidung für eine vorgesehene Nutzung nicht zwingend zur entsprechenden Nutzung führt, weil keine Pflicht zur Nutzung des Bodens besteht (HOFMANN 1974). Durch diese infrastrukturellen Verbesserungen sollen günstige Voraussetzungen zur Lenkung wirtschaftlicher Aktivitäten auf das gesteckte Ziel hin geschaffen werden.

Die entsprechenden Projektierungen und Ausführungen gehören dann, wie oben angedeutet, in den Bereich der Nutzungsplanung, wo für den Grundeigentümer verbindliche Festlegungen getroffen werden.

Die zweite Feststellung lautet:

Die Kartierung von Brachland ist eine wesentliche Grundlage zur Bewältigung des Problems, sie löst es aber nicht. Es braucht Entscheide zu Handlungen als verbindliche Beschlüsse der politischen Entscheidungsträger im Rahmen der Richtplanung.

\section{Die Beschreibung der Wirkungen von Handlungen}

Entscheidungen in der Raumplanung beziehen sich auf Handlungen. Entscheidungssituationen lassen sich nach BEHN + VAUPEL (1982) wie folgt darstellen (Fig. 1).

Aus diesem Schema wird deutlich, wie bedeutsam für Entscheidungen eine korrekte Aufzeichnung der Konsequenzen (Wirkungen) von Handlungen ist. Das Prädikat «korrekt» heißt in diesem Zusammenhang, daß die Konsequenzen verschiedener Handlungsmöglichkeiten anhand desselben Satzes an räumlichen Merkmalen beschrieben werden müssen. Ansonsten ist ein Vergleich und damit eine Bewertung der Resultate nicht möglich. Durch die Einführung einer derartigen "Objektsprache» tritt wohl in einem gewissen Sinne eine Verkümmerung der Alltagssprache ein, doch ist eine Systematisierung im obigen Sinne eine unabdingbare Voraussetzung rationalen Entscheidens. Aus dieser Sicht sei eine Bemerkung zu den am Geographischen Institut der Universität Zürich laufenden Arbeiten im Themenbereich «Brachland» angebracht:

Im Wissen um die raschen Veränderungen des Erscheinungsbildes von Brachland liegt das Schwergewicht richtigerweise weniger darauf, Monographien zu Brach-Landschaften zu verfassen, sondern darauf, Methoden zur raschen Erfassung von Brachland und zur adäquaten Speicherung der Informationen zu entwickeln. Erst durch die rasche Verfügbarkeit gespeicherter Daten wird es möglich, Simulationen künftiger Zustände der Landschaft durchzuführen. Dadurch können die im Entscheidungsbaum von BEHN + VAUPEL enthaltenen "consequence branches» in der notwendigen Präzision beschrieben werden. Den Entscheidungsträgern werden auf diese Weise verständliche Entscheidungsgrundlagen geliefert. 
Fig. 1 Entscheidungsbaum

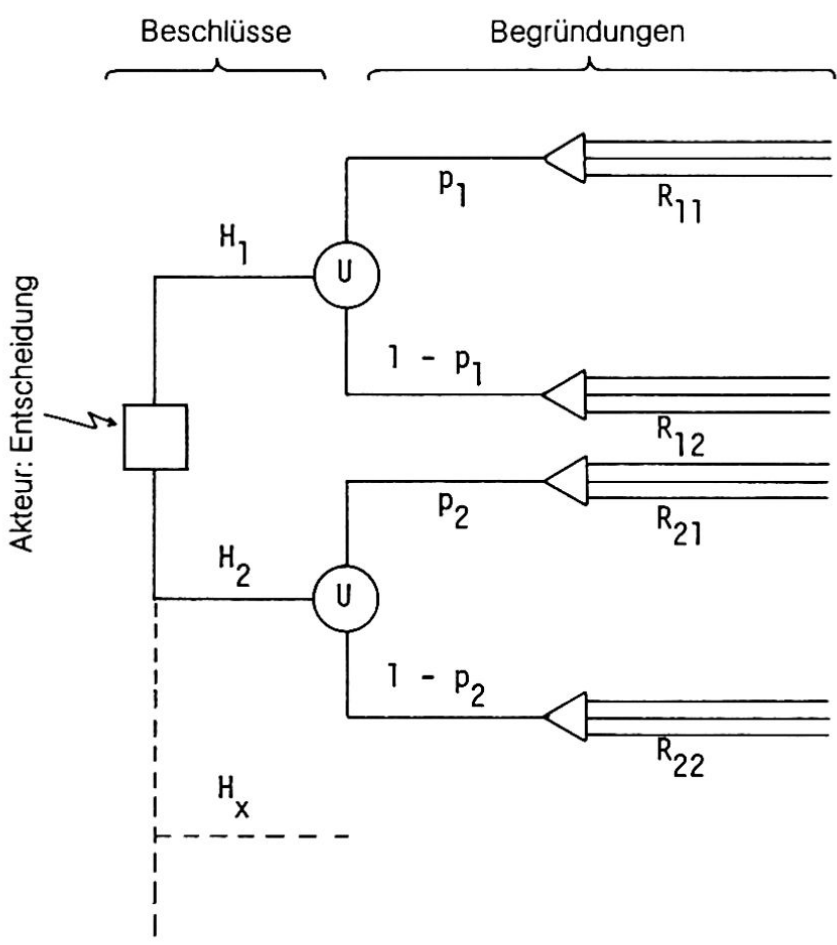

$\mathrm{H}$ : Mögliche Handlungen, Alternativen (Vorschläge zu Beschlüssen)

U: Ungewissheit (uncertainty node) (wegen äußerer Umstände)

p: Wahrscheinlichkeit, Glaubwürdigkeit des Eintreffens einer bestimmten Entwicklung

R: Resultate, räumliche Wirkungen (consequence branches)

Aus diesen Ausführungen wurde deutlich, daß bei der Richtplanung nach RPG nicht die einmalige Herstellung eines Planes, der für alle Zukunft sagt, was mit dem Brachland geschehen soll, im Vordergrund des Interesses steht. Aufgrund der Dynamik des Brachlandes (WALTHER 1982) ist eher angebracht, eine dauernde Beobachtung und Beurteilung der jeweiligen Lagen vorzunehmen und entsprechende Beschlüsse $\mathrm{zu}$ beantragen.

Folgende Fragen sind bei derartigen Beurteilungen etwa aufzuwerfen:

- Entwickelte sich die Brachlandausdehnung in der Weise, wie prognostiziert wurde?

- Sind die Wirkungen, die aufgrund von Handlungen erwartet wurden, auch eingetroffen?

- Haben sich Rahmenbedingungen im Umfeld des Brachlandes verändert?

- Müssen Änderungen in den Prioritäten der vorgesehenen Handlungen vorgenommen werden?
Aus diesen Ausführungen folgt die dritte Feststellung: Durch eine systematische Ordnung der Begriffe und der darauf aufbauenden konsequenten Beschreibung von Wirkungen werden die Voraussetzungen zur rationalen Bearbeitung der Brachlandprobleme im Rahmen der Richtplanung geschaffen.

\section{Schlussbemerkungen}

Es ging in diesem knapp gehaltenen Artikel darum, die Grundzüge der Richtplanung nach dem Bundesgesetz über die Raumplanung darzulegen und zu zeigen, $\mathrm{da} B$ für die Kantone, in denen Brachflächen in bedeutendem Umfang auftreten, ihre Bearbeitung im Rahmen der Richtplanung zwingend ist. Als wesentliche Voraussetzung einer sachgerechten Behandlung ist eine zweckdienliche Aufarbeitung der Grundlagen, gemäß den Ansätzen, die am Geographischen Institut der Universität Zürich unter Leitung von Prof. H.Haefner entwickelt wurden, hervorgehoben worden.

\section{Literaturverzeichnis}

BEHN, R., VAUPEL, J. (1982): Quick Analysis for Busy Decision Makers. Basic Books, New York.

Bundesgesetz über die Raumplanung (RPG) vom 22.Juni 1979

HOFMANN, M. (1974): Die Pflicht zur Nutzung des Bodens. Juris, Zürich.

SCHERRER, H.U., TRACHSLER, H., GRESCH, P. (1980): Vorschläge zur Kartierung und raumplanerischen Behandlung von Brachflächen. Eidg. Anstalt forstl. Versuchswes., Bericht Nr. 207, $20 \mathrm{ff}$.

SURBER, E., GRESCH, P., PFISTER, F. (1979): Die Kartierung von Brachland. Eidg. Anstalt forstl. Versuchwes. Bericht Nr. 199. Verordnung über die Raumplanung (VO RPG) vom 26. August 1981.

WALTHER, P., GRESCH, P., HAEFNER, H. (1982): Die Brachlandentwicklung im Obergoms 1970-1980. In: Geographica Helvetica, Nr. 1/82, 3-14. 